\title{
Editorial
}

Journal of Innate

Immunity

Published online: November 9, 2010

DOI: $\underline{10.1159 / 000322064}$

\section{Hemostasis in Invertebrates and Vertebrates: An Evolutionary Excursion}

\author{
Heiko Herwald ${ }^{a}$ Ulrich Theopold ${ }^{b}$ \\ a Department of Clinical Sciences, Biomedical Center, Lund University, Lund, and \\ ${ }^{b}$ Department of Molecular Biology and Functional Genomics, Stockholm University, Stockholm, Sweden
}

In 1964, Jack Levin and Frederik Barry Bang described a highly sensitive system to detect bacterial endotoxins (lipopolysaccharides; LPS): when mixed with a blood cell lysate from horseshoe crabs (Limulus), LPS-containing solutions immediately and very efficiently induced coagulation [1]. Named after its source of origin, the Limulus amebocyte was soon used routinely to detect whether solutions were free of bacterial contaminants. Due to the subsequent work described in this issue (Cerenius and Söderhäll [2]) we now understand that the coagulation of horseshoe crab is an intricate part of this spineless creature's innate immune system.

This is just one example of many showing that hemostasis is part of the host response to infection, and today we only start to comprehend how both systems are closely interweaved with each other. In this issue of the Journal of Innate Immunity, we therefore aim to present the state of the art in this area of exciting research. To this end, this issue will consist of 5 reviews and 2 original articles dealing with invertebrate and vertebrate clotting and their contribution to innate immunity.

The first review in this series of articles is an update on the coagulation cascade in the vertebrate line. Russell Doolittle [3] points out how clotting contributes to inflammatory reactions and visits some of the earliest

\section{KARGER}

Fax +4161306 1234

E-Mail karger@karger.ch

www.karger.com
(C) 2010 S. Karger AG, Basel

$1662-811 \mathrm{X} / 11 / 0031-0001 \$ 38.00 / 0$

Accessible online at:

www.karger.com/jin members of the evolutionary line that led to vertebrates. His article is a fascinating journey of important evolutionary aspects in hemostasis.

Despite the fact that fibrinogen-related proteins can be found in a wide range of invertebrates, they appear more relevant for immunity than clotting itself in most such cases as pointed out by Hanington and Zhang [4]. The authors propose that a function in clotting may in fact be a derived trait and that the original function for fibrinogen-like proteins may be within immunity. Even in vertebrates, fibrinogen domain-containing ficolins act as pattern recognition molecules. Similarly, invertebrate fibrinogen domains on their own or in combination with immunoglobulin domains can act as receptors for microbial and allorecognition. These domains have been shown to contribute to immunity, for example, in snails that are hosts for Schistosoma and may diversify somatically possibly via the proliferation of blood cells.

The article by Cerenius and Söderhäll [2] gives us a better understanding of the molecular mechanisms in some selected invertebrates whose clotting systems are best characterized. These include, for instance, the aforementioned horseshoe crabs, as well as crustaceans and insects where clotting had been described microscopically early on and molecular data are emerging. They also 
discuss emerging invertebrate models which may shed additional light on the evolution of clotting systems.

Eleftherianos and Revenis [5] provide us with insight into the phenoloxidase-activating system, the little or - in some cases - big brother of clotting, and describe the common mechanisms that have been observed in the 2 systems. The authors refer to recent work on insect clotting, reporting that the factors involved vary widely in their level of conservation. These findings are then discussed in an ecological and evolutionary context.

Loof et al. [6] discuss in their article differences and similarities seen in vertebrate and invertebrate hemostasis. The reader will not only learn about some surprising common principles in the molecular mechanisms of the clot's immune function in both living forms, such as the use of transglutaminase, but they will also read about important evolutionary differences in the invertebrate and vertebrate coagulation cascades.

Interestingly, the evolutionary variability of clotting systems is also reflected in the diversity of hemostatic and anti-inflammatory mechanisms that arthropods employ, for instance, while feeding on vertebrate blood. Ben J. Mans [7] sums up our knowledge on these immune-modulatory factors and provides some surprising insights into their evolution. In several cases the same host effector mechanisms appear to be targeted by different factors providing an exciting example of convergent evolution.

These reviews are followed by 2 research papers. Ferrandon and Matt [8] have characterized thioester-containing proteins (TEPs) from Drosophila, which show similarities with vertebrate complement factors. Despite their expression in immune tissues, Drosophila TEPs did not show a detectable role in immunity. This was somewhat surprising since the mosquito homologues contribute to immunity [9] and genome-wide scans have detected signals for positive selection in TEPs [10].

Hyrsl et al. [11] strengthen the idea that clotting is relevant for immunity and provide evidence that lipid mediators regulate clotting even in insects. This last finding may be yet another example of convergent evolution of the clotting system, which is a theme found in several contributions of this issue. Thus, it appears that clotting systems are on the one hand quite versatile and that this is paralleled by their parasites' virulence factors and by immune-modulatory factors of blood-feeding arthropods. On the other hand, similarities are also observed: certain protein domains and whole proteins contribute to clotting in phylogenetically quite distant organisms. Gene- and genome-wide scans have started to shed light on the underlying evolutionary forces and have provided evidence of both stabilizing and positive selection [11], which is very much in line with the dual function of clotting in wound closure and immunity.

With this collection of articles, the reader will be updated with the most current developments in this exciting field of research. Notably, evidence is accumulating that hemostasis and innate immunity act in close concert. However, we still see the tip of the iceberg and much more work is required to understand the complex interplay of both systems.

\section{References}

$>1$ Levin J, Bang FB: The role of endotoxin in the extracellular coagulation of Limulus blood. Bull Johns Hopkins Hosp 1964;115:265-274.

$>2$ Cerenius L, Söderhäll K: Coagulation in invertebrates. J Innate Immun 2011;3:3-8.

$>3$ Doolittle R: Coagulation in vertebrates with a focus on evolution and inflammation. J Innate Immun 2011;3:9-16.

4 Hanington PC, Zhang SM: The primary role of fibrinogen-related proteins in invertebrates is defense, not coagulation. J Innate Immun 2011;3:17-27
5 Eleftherianos I, Revenis C: Role and importance of phenoloxidase in insect hemostasis. J Innate Immun 2011;3:28-33.

6 Loof TG, Schmidt O, Herwald H, Theopold $\mathrm{U}$ : Coagulation systems of invertebrates and vertebrates and their roles in innate immunity: the same side of two coins? J Innate Immun 2011;3:34-40.

7 Mans BJ: Evolution of vertebrate hemostatic and inflammatory control mechanisms in blood-feeding arthropods. J Innate Immun 2011;3:41-45.

$>8$ Bou Aoun R, Hetru C, Troxler L, Doucet D, Ferrandon D, Matt N: Analysis of thioester containing proteins during the innate immune response of Drosophila melanogaster. J Innate Immun 2011;3:52-64.
-9 Blandin SA, Wang-Sattler R, Lamacchia M, Gagneur J, Lycett G, Ning Y, Levashina EA, Steinmetz LM: Dissecting the genetic basis of resistance to malaria parasites in Anopheles gambiae. Science 2009;326:147-150.

-10 Sackton TB, Lazzaro BP, Schlenke TA, Evans JD, Hultmark D, Clark AG: Dynamic evolution of the innate immune system in Drosophila. Nat Genet 2007;39:1461-1468.

11 Hyrsl P, Dobes P, Wang Z, Hauling T, Wilhelmsson C, Theopold U: Clotting factors and eicosanoids protect against nematode infections. J Innate Immun 2011;3:65-70. 